\title{
Vibrations of Cylindrical Shell Structures Filled With Layered Viscoelastic Material
}

\author{
Ismoil Safarov ${ }^{1,2}$, Muhsin Teshaev ${ }^{2,3,4^{*}}$, Abdurakhim Marasulov ${ }^{5}$, Tokhir Jurayev ${ }^{4}$, and \\ Bahodir Raxmonov ${ }^{6}$ \\ ${ }^{1}$ Tashkent Institute of Chemical Technology, Tashkent, Uzbekistan \\ ${ }^{2}$ Bukhara engineering-technological institute, Bukhara,_Uzbekistan \\ ${ }^{3}$ Bukhara branch of Institute of Mathematics AS RUz, Bukhara, Uzbekistan \\ ${ }^{4}$ Bukhara branch of the Tashkent Institute of Irrigation and Agricultural Mechanization \\ Engineers \\ ${ }^{5}$ Kazax-Turk International University, Kazakhstan \\ ${ }^{6}$ Urgench State University, Urgench, Uzbekistan
}

\begin{abstract}
A thin-walled shell and a thick-walled mass (cylinder) in contact with it, made of a different material, are structural elements of many machines, apparatus, and structures. The paper considers forced steady-state vibrations of cylindrical shell structures filled with a layered viscoelastic material. The study aims to determine the damping properties of vibrations of a structurally inhomogeneous cylindrical mechanical system under the influence of harmonic loads. The dynamic stress-strain state of a three-layer cylindrical shell filled with a viscoelastic material under the action of internal time-harmonic pressure is investigated. The oscillatory processes of the filler and the bonded shell satisfy the Lamé equations. At the contact between the shell and the filler, the rigid contact conditions are satisfied. Dependences between stresses and strains for a linear viscoelastic material are presented in the form of the BoltzmannVoltaire integral. The method of separation of variables, the method of the theory of potential functions (special functions), and the Gauss method are used to solve this problem. Based on the analysis of the numerical results, it was found that the dependence of the resonant amplitude of the shell displacements on the viscous properties of the filler is $12-15 \%$. Analysis of the results obtained shows that the study of vibrations of shells containing fillers according to the rod theory will lead to rather large erroneous results (up to $20 \%$ ).
\end{abstract}

\section{Introduction}

Acceleration of scientific and technological progress, intensive development of the national economy will lead to the need to create new systems and technologies that should work in wide ranges of speeds, temperatures, pressures, and loads. At the same time, they must be convenient for operation, comfortable for humans, environmentally friendly, silent, etc. Such systems, first of all, include a variety of vehicles: airplanes, helicopters, automobiles,

*Corresponding author: muhsin_5@mail.ru 
etc. For such systems, one of the topical aspects is the problem of suppressing vibrations arising under the influence of external loads [1-4].

A thin-walled shell and a thick-walled mass (cylinder) made of different material in contact with its inner or outer surfaces are structural elements in many machines, apparatus, and structures $[5,6]$. The works $[7,8,9]$ are devoted to calculating the stress-strain state of the dynamic processes of thin-walled shells and the elastic or viscoelastic filler contained in them. In addition, these issues affect the literature on solid fuel engines to one degree or another. For example, the design of solid-propellant rocket engine structures has led to a long-term study of a structural system consisting of a hollow viscoelastic cylinder enclosed in a thin body (viscoelastic shell) $[10,11]$. A large number of problems of axisymmetric configuration under plane deformation have been formulated and solved. In this case, the quasi-static linear theory of viscoelasticity was usually used.

The aim of the work is to study the damping properties of vibrations of a structurally inhomogeneous mechanical system under the influence of local loads.

To study the damping properties of vibrations of a mechanical system, taking into account the influence of the geometric and physical-mechanical parameters of the shell, the method of separated variables, the method of the theory of potential functions (special functions), and the Gauss method are used.

\section{Methods}

\subsection{Formulation of the problem}

Consider a long viscoelastic multilayer cylinder with a circular cavity of constant radius a and with a radius of the outer surface $b$ (total radius), along which the cylinder is attached to an elastic (or viscoelastic) shell (Figure 1). Some layers (elements) of the cylinder can be massless. In this case, they are characterized by stiffness coefficients. The material of the multilayer cylinder is a typical highly filled polymer, the physical and mechanical characteristics of which are determined by the nature of the binder, the adhesion of the binder to the multilayer filler. The system "cylinder - body (shell)" is subject to timevarying uniform normal (vibration) pressure.

The dynamic stress state of a three-layer cylindrical shell filled with a viscoelastic material under the action of internal dynamic pressure is considered.
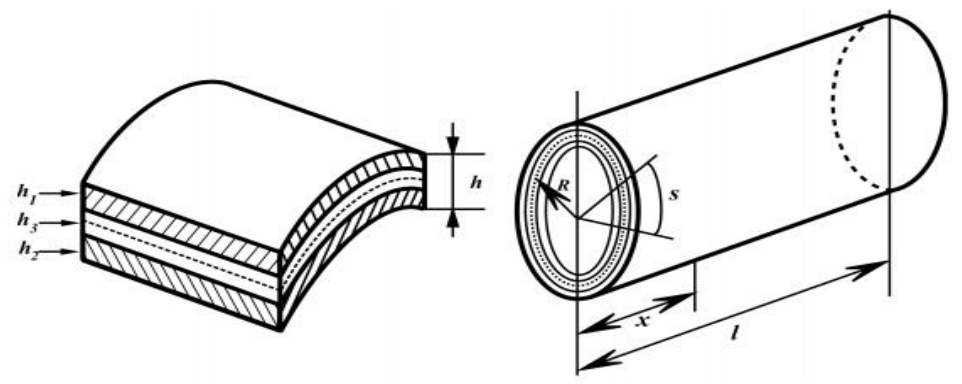

Fig.1. Three-layer construction ( $h_{1}$ - first layer, $h_{2}$ - second layer, 3- filler)

The equations of small vibrations of a layered cylinder have the form [12]

$$
\tilde{\mu}_{\mathrm{K}} \nabla^{2} \vec{u}+\left(\tilde{\lambda}_{\mathrm{K}}+\tilde{\mu}_{\mathrm{K}}\right) \operatorname{graddiv} \vec{u}=\rho_{\mathrm{K}} \frac{\partial^{2} \vec{u}}{\partial t^{2}},(k=1,2,3 \ldots . . N-1)
$$


If a $\mathrm{k}=\mathrm{N}$, then (1) describes the equation of motion of the shell. Here $\overrightarrow{u_{k}}\left(u_{r k}, u_{\theta k}, u_{x k}\right)$ and $\overrightarrow{u_{N}}(w, v, u)$ - displacement vectors, is the density of the $\mathrm{k}$-th material.

The boundary conditions at the contact between the layers are

$$
\begin{gathered}
\sigma_{r}^{i}=\sigma_{r}^{i+1} ; \tau_{r x}^{i}=\tau_{r x}^{i+1} ; \tau_{r \varphi}^{i}=\tau_{r \varphi}^{i+1} \\
u_{r}^{i}=u_{r}^{i+1} ; u_{\theta}^{i}=u_{\theta}^{i+1} ; u_{x}^{i}=u_{x}^{i+1}
\end{gathered}
$$

where index $i$ matches the layer number $i=1,2,3, \ldots N ; \quad r=r_{j}$. The layers are numbered starting with the outer one. If between $i$ - oh and $i+1$ - the layer contains a massless cylinder, then the boundary conditions take the form (at $\left.r=r_{j}\right)$ :

$$
\begin{gathered}
\sigma_{r r i}=k_{r}\left(u_{r i}-u_{r(i+1)}\right), \tau_{r \theta i}=k_{\theta}\left(u_{\theta i}-u_{\theta(i+1)}\right) \\
\tau_{r x i}=k_{x}\left(u_{x i}-u_{x(i+1)}\right)
\end{gathered}
$$

where $k_{r}, k_{\theta}, k_{x}$ are interaction coefficients. As an example, consider a cylindrical filler $r=R_{0}$ and $r=R_{1}$, bonded sheath.

In the structure (figure 1$)$, the left end $(\mathrm{z}=0)$ is rigidly clamped, and the right end $(\mathrm{z}=$ $l$ ) is free. When studying forced vibrations, it is assumed that a load is applied to the inner surface

$$
\begin{gathered}
r=R_{0}: \sigma_{r x}=\sigma_{r \varphi}=0, \sigma_{r r}=Q_{0}(t) \\
Q_{0}(t)=P_{0} e^{-i p t}, \\
r=R_{b}: \sigma_{r}=\tau_{r \varphi}=\tau_{r x}=0
\end{gathered}
$$

where $p$ is the frequency of external influences; $\mathrm{P}_{0}$ is the amplitude of external influences; $R_{0}$ and $R_{b}$ are inner and outer radii of the cylinder under consideration.

As an example, consider the dynamic stressed state of a half-cylinder under the action of internal pressure, i.e., consider the change in the resonance region depending on the parameters at different viscosities. The relationship between stresses and strains for a linear viscoelastic material can be represented as

$$
\sigma_{i j \mathrm{\kappa}}=\lambda_{k}\left(1-R_{\lambda k}\right) \theta_{k} \delta_{i j}+2 \mu_{k}\left(1-R_{\mu \kappa}\right) \varepsilon_{i j \kappa}
$$

Here, $\lambda_{\kappa}, \mu_{\kappa}$ are operator modulus of elasticity $\kappa$ - th element, $\varepsilon_{i j \mathrm{~K}}$ is strain tensor elements $\kappa$ th element, $\theta$ is volumetric deformation

$$
R_{\lambda} f(t)=\int_{0}^{t} R_{\lambda k}(t-\tau) f(\tau) d \tau ; R_{\mu} f(t)=\int_{0}^{t} R_{\mu k}(t-\tau) f(\tau) d \tau
$$

$f(t)$ is time derivative; $R_{\lambda_{\kappa}}(t-\tau), R_{\mu \kappa}(t-\tau)$ are relaxation kernels; $\lambda_{0 \kappa}, \mu_{0 \kappa}$ are instantaneous coefficients of the modulus of elasticity $(\mathrm{k}=1, \ldots, \mathrm{N})$ [13].

The equations of motion of this structure satisfy the Lamé equations in a cylindrical coordinate system and are solved in the displacement potentials [14]. Let us introduce three scalar potential functions $\varphi_{k}, \psi_{k}, \chi_{k}$, which are solutions of equations [14].

$$
\begin{aligned}
& \nabla^{2} \varphi_{\kappa}-\int_{-\infty}^{t} R_{l \kappa}(t-\tau) \nabla^{2} \varphi_{\kappa}(\tau) d t=\frac{1}{c_{l 0 \kappa}^{2}} \frac{\partial^{2} \varphi_{\kappa}}{\partial t^{2}}, \\
& \nabla^{2} \psi_{\kappa}-\int_{-\infty}^{t} R_{t k}(t-\tau) \nabla^{2} \psi_{\kappa}(\tau) d t=\frac{1}{c_{t 0 \kappa}^{2}} \frac{\partial^{2} \psi_{\kappa}}{\partial t^{2}}
\end{aligned}
$$




$$
\nabla^{2} \chi_{\kappa}-\int_{-\infty}^{t} R_{t \kappa}(t-\tau) \nabla^{2} \chi_{\kappa}(\tau) d t=\frac{1}{c_{t 0 \kappa}^{2}} \frac{\partial^{2} \chi_{\kappa}}{\partial t^{2}}
$$

where $c_{l 0 \kappa}^{2}=\frac{\lambda_{0 \kappa}+2 \mu_{0 \kappa}}{\rho_{\mathrm{K}}}, c_{t 0 \kappa}^{2}=\mu_{0} / \rho_{\mathrm{K}}$ is the velocity of propagation of longitudinal and transverse waves, respectively.

\subsection{Solution methods}

The solution of integro-differential equations (6) is sought in the form

$$
\begin{array}{r}
\varphi_{k}(r, \theta, z, t)=\sum_{n=0}^{\infty} \varphi_{k n}\left(\alpha_{k} r\right)\left\{\begin{array}{c}
\cos n \theta \\
-\sin n \theta
\end{array}\right\} \cos \left(\gamma_{k} x+\delta\right) e^{i p t} \\
\psi_{k}(r, \theta, z, t)=\sum_{n=0}^{\infty} \psi_{k n}\left(\beta_{k} r\right)\left\{\begin{array}{c}
\sin n \theta \\
-\cos n \theta
\end{array}\right\} \sin \left(\gamma_{k} x+\delta\right) e^{i p t}, \\
\chi_{k}(r, \theta, z, t)=\sum_{n=0}^{\infty} \chi_{k n}\left(\beta_{k} r\right)\left\{\begin{array}{c}
\cos n \theta \\
-\sin n \theta
\end{array}\right\} \sin \left(\gamma_{k} x+\delta\right) e^{i p t}
\end{array}
$$

where $\varphi_{n}, \psi_{n}, \chi_{n}$ are amplitudes of longitudinal and transverse potentials, $\alpha_{k}$ and $\beta_{k}$ are wave numbers; $\gamma_{k}$ is complex wavenumber, $\delta_{k}$ is phase shift, $p$ is frequency of external loads.

Substituting (7) into (6), we obtain the Helmholtz equations [15]. Their solutions are expressed through the Bessel functions $J_{n}$ and $N_{n}$ :

$$
\begin{array}{r}
\varphi_{n k}=\left[A_{1 n k} J_{n}\left(\alpha_{l k} r\right)+A_{2 n k} N_{n}\left(\alpha_{l k} r\right)\right] ; \\
\psi_{n k}=\left[B_{1 n k} J_{n}\left(\beta_{l k} r\right)+B_{2 n k} N_{n}\left(\beta_{l k} r\right)\right] ; \\
\chi_{n k}=\left[C_{1 n k} J_{n}\left(\beta_{l k} r\right)+C_{2 n k} N_{n}\left(\beta_{l k} r\right)\right],
\end{array}
$$

where $A_{1 n k}, A_{2 n k}, B_{1 n k}, B_{2 n k}, C_{1 n k}, C_{2 n k}$ are arbitrary constants, $\alpha_{l k}, \beta_{l k}$ are wave numbers of longitudinal and shear waves, which are complex quantities,

$$
\begin{gathered}
\alpha_{l k}^{2}=\mu_{k l}^{2}-\gamma_{k}^{2}, \beta_{t k}^{2}=\mu_{k t}^{2}-\gamma_{k}^{2}, \mu_{k l}=p / c_{l k} \Gamma_{k 1}, \mu_{k t}=p / c_{t k} \Gamma_{k 1}, \\
\Gamma_{k 1}=1-\Gamma_{k 1}^{C}(p)-i \Gamma_{k 1}^{S}(p) ; \Gamma_{k 2}=1-\Gamma_{k 2}^{C}(p)-i \Gamma_{k 2}^{S}(p), \\
\Gamma_{k 1}^{c}(p)=\int_{0}^{\infty} R_{k 1}(\tau) \cos p \tau d \tau, \Gamma_{k 2}^{c}(p)=\int_{0}^{\infty} R_{k 2}(\tau) \cos p \tau d \tau, \\
\Gamma_{k 1}^{s}(p)=\int_{0}^{\infty} R_{k 1}(\tau) \sin p \tau d \tau, \Gamma_{k 2}^{S}(p)=\int_{0}^{\infty} R_{k 2}(\tau) \sin p \tau d \tau,
\end{gathered}
$$

the cosine and sine of the Fourier image of the relaxation kernels, respectively; $p$ is a real value. The calculations used the Koltunov-Rzhanitsyn three-parameter relaxation kernel: $R_{k}(t)=A_{k} e^{-\beta_{k} t} / t^{1-\alpha_{k}}$. Continuous and shear wave potentials $\varphi_{k}, \psi_{k}, \chi_{k}$ is related to the components of the displacement vector, in a cylindrical coordinate system, by dependencies [16]:

$$
\begin{aligned}
& u_{x k}=\frac{\partial \varphi_{k}}{\partial x}+\frac{1}{\mu_{t k}}\left(\mu_{t k}^{2} \chi_{k}+\frac{\partial^{2} \chi_{k}}{\partial x^{2}}\right) \\
& u_{\theta k}=\frac{\partial \varphi_{k}}{r \partial \theta}-\frac{\partial \psi_{k}}{\partial r}+\frac{1}{\mu_{t k}} \frac{\partial^{2} \chi_{k}}{r \partial \theta \partial x}
\end{aligned}
$$




$$
u_{r k}=\frac{\partial \varphi_{k}}{\partial r}+\frac{\partial \psi_{k}}{r \partial \theta}+\frac{1}{\mu_{t k}} \frac{\partial^{2} \chi_{k}}{r \partial x \partial r}
$$

In this case, the expressions for stresses and mixed are complex quantities. Actual stresses are determined by the following expressions:

$$
\operatorname{Re}\left[\left(\sigma_{r r}^{(R)}+i \sigma_{r r}^{(I)}\right) e^{i p t}\right]=\sigma_{r r}^{(R)} \cos p t-\sigma_{r r}^{(I)} \sin p t
$$

Suppose that a liquid or gas from the inner side acts on the structure with harmonic pressure. Then this pressure can be represented as

$$
p=\sum_{k=0}^{\infty} \sum_{n=0}^{\infty} P_{k} \sin \alpha_{k} x \cos n \theta e^{i p t} .
$$

If we consider a structure located on the xOy plane, then in the shell and filler, the radial displacement has the form:

$$
\begin{gathered}
w=\sum_{k=0}^{\infty} \sum_{n=0}^{\infty} W_{k n} \sin \alpha_{k} x \cos n \theta e^{i p t}, \\
u_{r}=\sum_{k=0}^{\infty} \sum_{n=0}^{\infty}\left\{\alpha_{k}\left[A_{1 k n} J_{n}\left(\gamma_{i k} r\right)+A_{2 k n} N_{n}\left(\gamma_{i k} r\right)\right]+\right. \\
\left.+\frac{\gamma_{i k}^{2}}{\mu_{i k}}\left[C_{1 k n} J_{n}\left(\gamma_{i k} r\right)+C_{2 k n} N_{n}\left(\gamma_{i k} r\right)\right]\right\} \sin \alpha_{k} x \cos n \theta e^{i p t}
\end{gathered}
$$

where $\gamma_{l k}^{2}=\mu_{l}^{2}-\alpha_{k}^{2}, \gamma_{t k}^{2}=\mu_{t}^{2}-\alpha_{k}^{2}, \mu_{l}=p / \bar{a}_{l} \mu_{t}=p / \bar{a}_{t}$.

If a $\gamma_{l k}^{2}<0, \gamma_{t k}^{2}<0$, then instead of $J_{n}(z), N_{n}(z)$ use accordingly $I_{n}(z), K_{n}(z)$.

Thus, for each layer of a cylindrical body (for an extended cylindrical layer) solutions can be written in terms of displacements in the following form:

$$
\begin{gathered}
u_{r}=\sum_{n=0}^{\infty}\left[F_{n} \frac{d H_{n}^{(1)}(\alpha r)}{d r}+D_{2 n} i \gamma_{p} H_{n+1}^{(1)}(\beta r)+M_{1 n} n H_{n}^{(1)}(\beta r) / r\right]\left(\begin{array}{c}
\cos n \theta \\
-\sin n \theta
\end{array}\right) \sin (\gamma x+\delta) e^{i p t} \\
u_{\theta}=\sum_{n=0}^{\infty}\left[-F_{n} n H_{n}^{(1)}(\alpha r) / r+D_{2 n} i \gamma_{p} H_{n+1}^{(1)}(\beta r)-M_{1 n} \frac{d H_{n}^{(1)}(\beta r)}{d r}\right]\left(\begin{array}{c}
\sin n \theta \\
\cos n \theta
\end{array}\right) \sin (\gamma x+\delta) e^{i p t},(9) \\
u_{z}=\sum_{n=0}^{\infty}\left[-F_{n} i \gamma_{p} H_{n+1}^{(1)}(\alpha r)-D_{2 n}\left[\frac{d H_{n+1}^{(1)}(\beta r)}{d r}+\frac{n+1}{r} H_{n+1}^{(1)}(\beta r)\right]\right]\left(\begin{array}{c}
\cos n \theta \\
-\sin n \theta
\end{array}\right) \cos (\gamma x+\delta) e^{i p t} .
\end{gathered}
$$

Displacement expressions (9) contain three arbitrary constants $F_{n}, D_{2 n}, M_{1 n}$ and satisfy the equations of motion (1) for all values of the frequency p. For a short spatial cylinder, the number of arbitrary constants will be six. If the obtained solutions in terms of potentials are expressed with the help of special Bessel and Neumann functions, and also the boundary conditions are used, then we obtain a system of inhomogeneous algebraic equations from 12 unknowns.

\section{Results and Discussion}

The resulting aggregate stresses are shown in Figures 2-6. The relationship between the amplitude of the shear stress and the frequency of the forced stress is shown in Figure 2, for different $\delta_{1} / \pi$ : 1)0.3; 2)0.2; 3) $0.1, R_{0} / R=0.5$ и $\gamma_{p}=0.33$. 


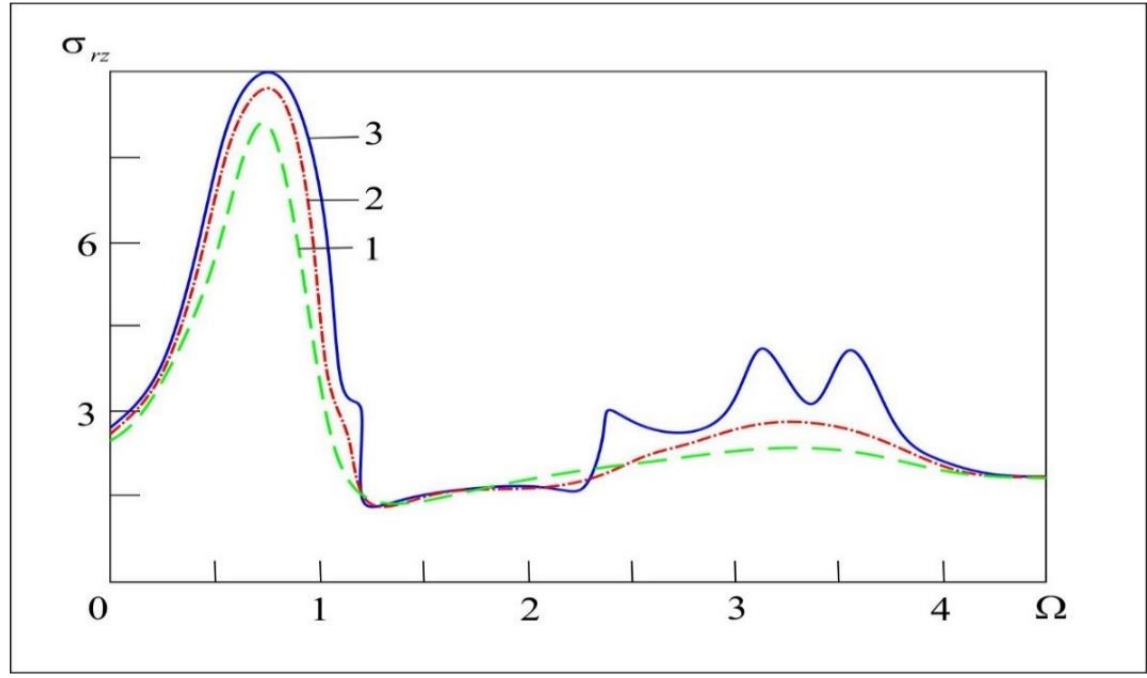

Fig. 2. Relationship between shear stress amplitude and forced stress frequency

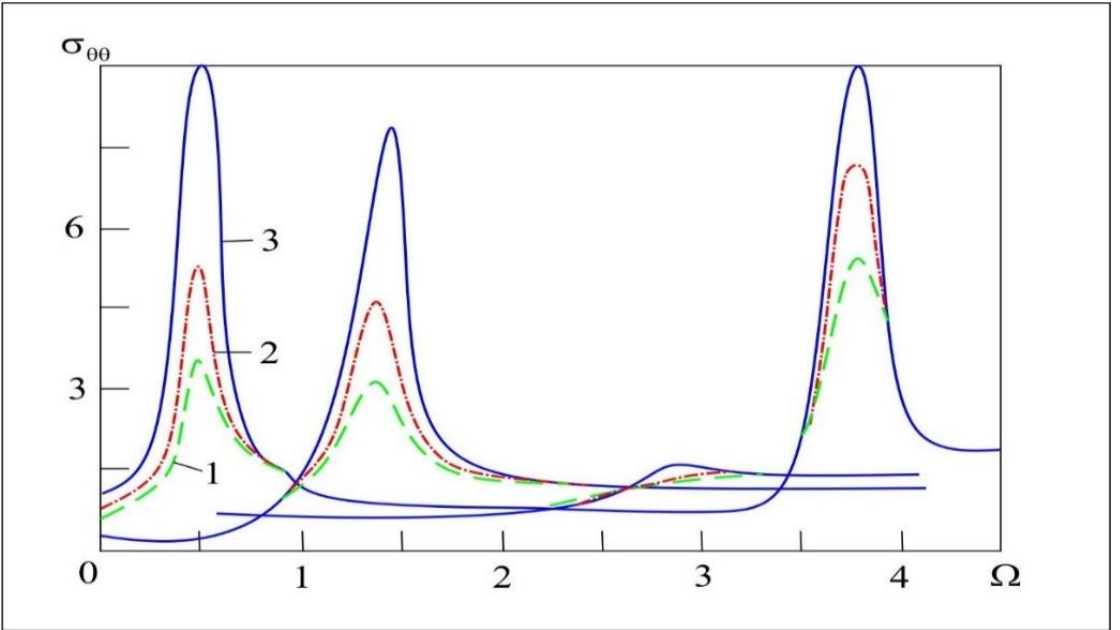

Fig.3. The relationship between the amplitude of the loop voltage and the frequency of forced voltages

Here, $\delta_{1}$ is logarithmic decrement, which is determined by expression: $\delta_{1}=2 \pi \ln \left|\omega_{I 1} / \omega_{R 1}\right|$. Figure 3 shows that with a decrease in the damping decrement coefficient, the corresponding amplitudefrequency characteristics increase. 


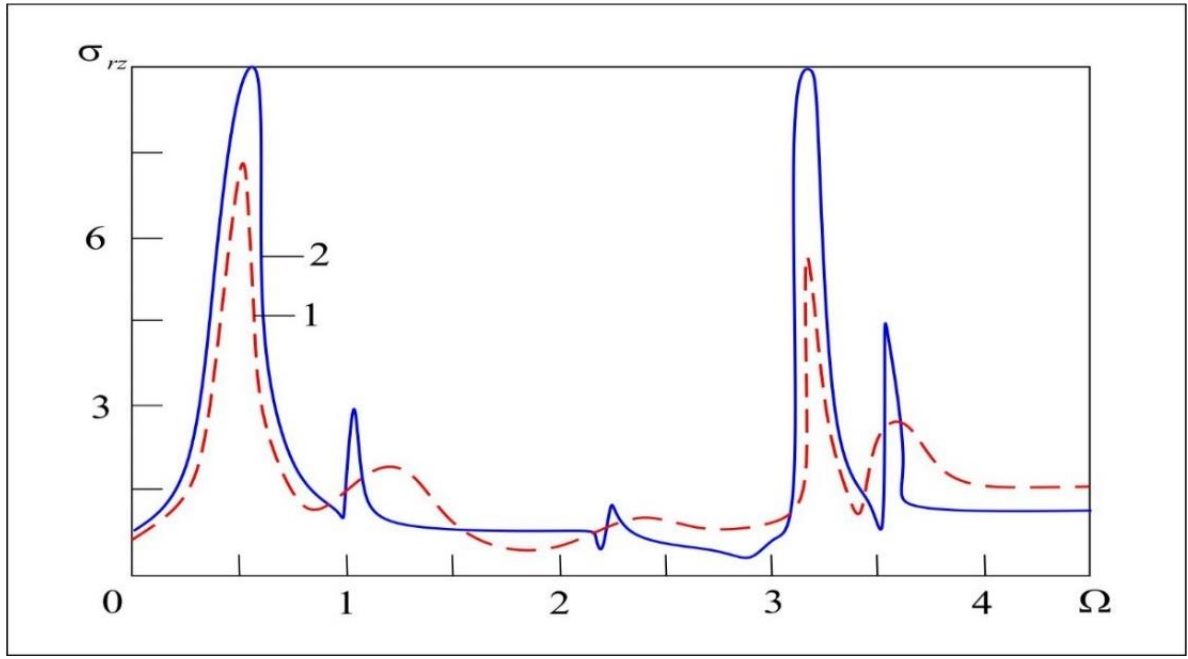

Fig.4. Relationship between the shear stress amplitude and the forced stress frequency

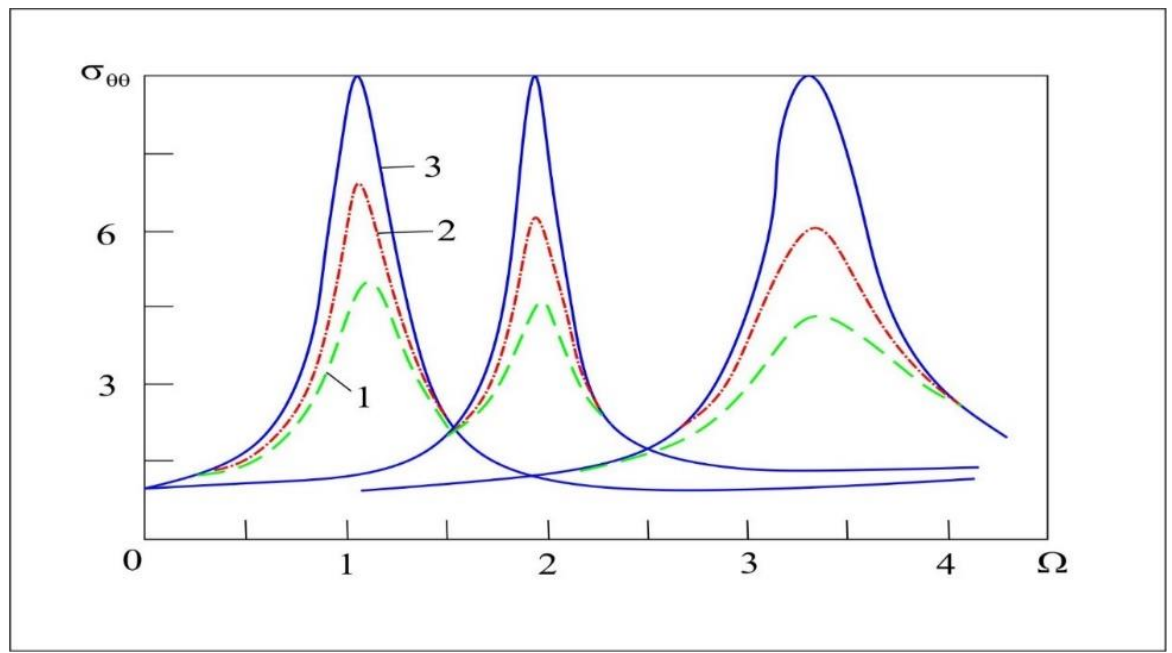

Fig.5. The relationship between the amplitude of the loop voltage and the frequency of forced voltages

Figure 4 shows the change in the first resonance peak depending on the frequency for different values of the ratio of the radii ( $\mathrm{R}$ is the outer radius, $\mathrm{R}_{0}{ }^{-}$inner radius of the filler) of the cylindrical body. The considered mechanical system is dissipatively homogeneous $[17,18]$. It is seen that with an increase in the ratio of the radii, the corresponding amplitude-frequency characteristics of the loop voltage decrease.

In the second resonance peak $\gamma_{p} R_{0}=0.70$. The third resonance peak shows the amplitude-frequency characteristics of the loop voltage at different values of the damping decrement [19].

Figure 4 shows the change in the amplitude of the tangential stresses of the aggregate depending on the frequency for different values of the rheological parameters of the material. (1. $\mathrm{A}=0.078 ; 2 . \mathrm{A}=0.036$ ). The considered mechanical system is dissipatively inhomogeneous (for a shell $\mathrm{R}_{0}=0$ ) [20,21]. It can be seen that the amplitudes of displacements, depending on the frequency, take on their maximum value at different 
values of the natural vibrations of mechanical systems. Figure 5 shows the changes in the amplitude of the contour stresses in the shell depending on the dimensionless frequencies, i.e., the resonance curves are presented. Curves 1,2,3 indicate the stresses between the cylindrical aggregate and the contact surface at the point $x=l / 2$ at different values of the damping decrement: 1)0.3; 2)0.2; 3) 0.1 and at $\gamma_{p}=0.33$.

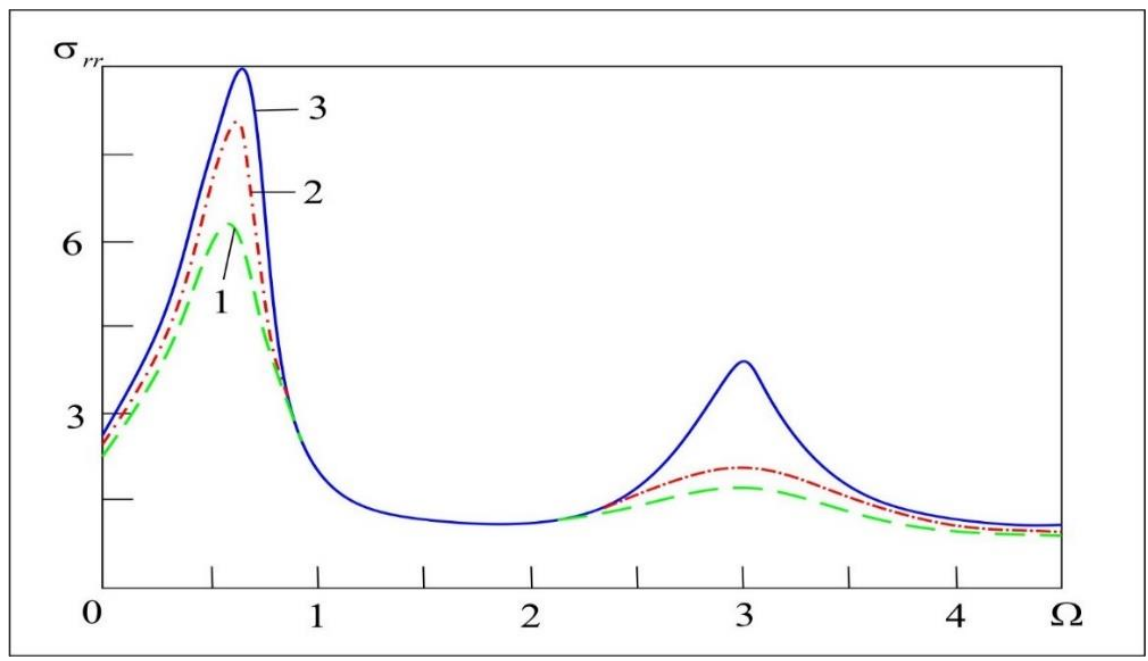

Fig.6. The relationship between the amplitude of the radial stress and the frequency of forced stresses

The first resonance peak was obtained at $h / R=0.95$, the second - at $h / R=0.75$, and the third - at $h / R=0.5$. It is seen that at $\delta_{1} / \pi=0.1(A=0.078, \alpha=0.5)$, even if the maximum values of the loop stresses are reduced by $20-25 \%$, they retain their intensity. Exactly the same - the same tendency persists for radial stresses..

Figure 6 shows the change in the amplitude of the radial stresses of the filler depending on the frequency for different values of the rheological parameters of the shell and filler material $(1 . \mathrm{A}=0.078 ; 2 . \mathrm{A}=0.036, \mathrm{~A}=0.018)$. The considered mechanical system is dissipatively homogeneous. It was found that the amplitudes of displacements, their maximum value are taken at the first resonant frequency of oscillations of mechanical systems. For a dissipatively inhomogeneous mechanical system, the displacement amplitudes take their maximum value at the second or third resonance frequencies.

The obtained numerical results were compared with the numerical results obtained from the analytical solution $[22,23]$ for the same values of parameters. The difference in results was up to $12 \%$.

The research results can also be used to develop a new design for drying cotton seeds $[24,25]$, as well as in improving the energy efficiency and reliability of the power supply $[26,27]$.

Here it was also possible to consider the control of body vibrations by introducing servo constraints [28-30], which will be considered later.

\section{Conclusions}

Based on the analysis of numerical results, it was established:

1. The dependence of the resonant amplitude of displacements of the shell on the viscous properties of the filler is $12-15 \%$. 
2. Analysis of the results obtained shows that studies of vibrations of shells containing a filler, according to the rod theory, will lead to rather large erroneous results (up to $20 \%$ ).

\section{Reference}

1. Ilgamov M A, Ivanov V A and Gulin B A Strength, stability and dynamics of shells with elastic core (Science) p. 331 (1977)

2. Vlasov V Z and Leontiev N N 1960 Beams, slabs, shells on an elastic base "research institutes (Moscow: Nauka) p. 491. (1960).

3. Starovoitov E I Viscoelastoplastic layered plates and shells (Gomel) p. 344 (2002)

4. Pleskachevsky Yu M, Starovoitov E I and Leonenko D V, Mechanics of 3-layer rods and Plates associated with an elastic foundation (M: FIZMATLIT). (2003)

5. Alexey A, Semenov. Model of deformation stiffened orthotropic shells under dynamic loading, Journal of Siberian Federal University. Mathematics and Physic 9 (4) pp. 485-497

6. Bosyakov S M and Chzhiwei V 2011 Analysis of free vibrations of cylindrical shells made of fiberglass with Navier boundary conditions, Mechanics of machines, mechanisms and materials (3) pp. 24-27. (2011)

7. Latifov F.S, Seyfullaev F.A and Alyev Sh Sh, Free vibrations reinforced by transverse ribs of an anisotropic cylindrical shell made of fiberglass with a liquid flowing in it, Applied mechanics and technical physics Vol 57 № 4 pp. 158-162

8. Seyfullayev A.I and Novruzova K.A, Oscillations of longitudinally reinforced orthotropic cylindrical shell filled with a viscous fluid, Eastern-European Journal of Enterprise Technologies 3 (75) pp. 29-33. (2015)

9. Bolotin V V and Beginners Yu N, Mechanics of multilayer structures, (M: Mechanical Engineering) p. 375

10. Lugovoi P Z, Meish V F and Stantzel E A Nonstationary dynamics of non-uniform shell structures (K: Knowledge) p. 538. (2005)

11. Meish V.F and Latanskaya L.A, Axisymmetric vibrations of three-layer cylindrical shells with piecewise-homogeneous filler with non-stationary loaded,Bulletin of Donetsk University. Series A: Natural Sciences. Science journal. - Donetsk: DonNU VIP 1 pp. 161-164. (2008)

12. Lugovoi P Z, 2001 Dynamics of thin-walled structures under non-stationary loads, Applied Mechanics 37 (5) pp. 44-73 (2001).

13. Koltunov M A, Karimov A I and Mavlyanov T 1981 One method for solving the problem of the dynamical stability of thin-walled viscoelastic structures, Journal Mechanics of Composite Materials 16 (5) September pp. 591-595 (DOI: 10.1007/BF00610188)

14. Mavlyanov $\mathrm{T}$ and Koltunov M A,Vibrations of viscoelastic orthotropic shells in various media, Journal Polymer Mechanics, 11 (5) September pp. 710-717 DOI: 10.1007/BF00859647. (1975).

15. Noor A K and Burton A K, Computational model for high temperature multilayered composite plates and shells Applied Mech. Rev 45 (10) pp. 419-446. (1992)

16. Teshaev M K, Safarov I I, Kuldashov N U, Ishmamatov M R and Ruziev T R, On the Distribution of Free Waves on the Surface of a Viscoelastic Cylindrical Cavity Journal of Vibrational Engineering and Technologies 8(4) pp 579-585

17. Safarov I I, Teshaev M X, Toshmatov E, Boltaev Z I and Homidov F F, Torsional vibrations of a cylindrical shell in a linear viscoelastic medium IOP Conference Series: Materials Science and Engineering 883 (1) 012190. (2020) 
18. Mirsaidov M M, Safarov I.I, Teshaev M.K and Boltayev Z I, Dynamics of structural Inhomogeneous coaxial-multi-layered systems cylinder-shells, Journal of Physics: Conference Series. 1706 (1) 012033. (2020).

19. Teshaev M.Kh, Safarov I. I and Mirsaidov M. M, Oscillations of multilayer viscoelastic composite toroidal pipes, Journal of the Serbian Society for Computational Mechanics 13 (2) pp 104-115. (2019).

20. Mirsaidov M M, Safarov I I and Teshaev M X, Dynamic instability of vibrations of thin-wall composite curvorine viscoelastic tubes under the influence of pulse pressure 164 (14013) DOI: 10.1051/e3sconf/202016414013 (2020)

21. Boltaev Z.I, Safarov I.I and Razokov T, Natural vibrations of spherical inhomogeneity in a viscoelastic medium, International Journal of Scientific and Technology Research 9 (1) pp 3674-3680, (2020).

22. Durdiev D.K and Totieva Z.D, The problem of determining the one-dimensional kernel of viscoelasticity equation with a source of explosive type Journal of Inverse and Ill-Posed Problems 28 (1) pp 43-52, (2020)

23. Durdiev D K and Rahmonov A A, Inverse Problem for A System of IntegroDifferential Equations for SH Waves in A Visco-Elastic Porous Medium: Global Solvability, Theoretical and Mathematical Physics(Russian Federation) 195(3) pp 923-937, (2018).

24. Rahmonov X K, Improvement of Equipment and Technology of Drying of the Cotton Mass and its Technological Assessment on the Basis of its Thermal Properties International Journal of Advanced Research in Science, Engineering and Technology, (2019)

25. Rahmonov X K, Development of a New Design for Drying Cotton Seeds with Purpose of Efficient Use of Heat, International Journal of Advanced Research in Science, Engineering and Technology 74 May (www.ijarset.com) (2020)

26. Sadullayev N N, Safarov A B, Nematov S N and et al, Opportunities and Prospects for the Using Renewable Energy Sources in Bukhara Region, Appl Sol Energy 56 pp 291-300 https://doi.org/10.3103/S0003701X20040106. (2020)

27. Sadullaev N N, Mukhamedkhanov U T, Nematov SH N and Sayliev F O, Increasing Energy Efficiency and Reliability of Electric Supply of Low Power Consumers, International Journal of Engineering Trends and Technology 68 (12) pp 43-47 doi:10.14445/22315381/IJETT-V68I12P208. (2019).

28. Khusanov K, Stabilization of mechanical system with holonomic servoconstraints. IOP Conf Se:r Mater Sci Eng 883 012146. (2020)

29. Khusanov K, Stabilization of mechanical system with nonholonomic servoconstraints constraints. IOP Conf Ser: Mater Sci Eng 883012164 (2020)

30. Khusanov K, Equations of motion of mechanical systems with nonlinear nonholonomic servoconstraints. IOP Conf Ser: Mater Sci Eng 869 072021. (2020) 